\title{
1.5 X-RAY OBSERVATIONS OF THE CRAB NEBULA
}

\author{
EDWIN M. KELLOGG
}

American Science and Engineering, 11 Carleton Street, Cambridge, Mass. U.S.A.

\begin{abstract}
This paper is a review of the X-ray observations made on the Crab Nebula They include angular size, location, energy spectrum as high as $560 \mathrm{keV}$, upper limits on time variability and line emission, interstellar absorption and polarization. Some data on the X-ray spectrum of the pulsar NP0532 are included, but for details on the X-ray pulsar, see the paper by Rappaport (1971). The $\mathrm{X}$-ray luminosity versus radius of the Crab, compared with that of other known X-ray emitting supernova remnants is also discussed.
\end{abstract}

\section{Introduction}

The Crab Nebula has been observed in X-rays on more than thirty different occasions since 1964, using both sounding rocket and balloon experiments. The X-ray source in the Crab consists of a pulsed component, obviously associated with the radio and optical pulsar NP 0532, and a continuous emitter, which has a finite size of about 1-2 arc min. The continuous source emits about $90 \%$ of the X-ray power in the range $2-100 \mathrm{keV}$, which represents a large fraction of the total radiation from the Crab over all wavelengths.

Measurements of the angular structure have been very crude so far, allowing only approximate estimates of size, but no details on the X-ray brightness distribution. A good deal of data exist on the spectrum, which allow an extrapolation with reasonable confidence down through the frequency regions obscured by interstellar absorption to compare with the optical and radio data. This is perhaps the most useful result of the X-ray observations so far, for it allows comparison of the data with synchrotron models of the source over twelve decades in frequency.

Measurements of other parameters such as time variability, line emission, interstellar absorption, and polarization are still crude, not able to provide definitive answers about the nature of the Crab, but they are discussed here in order to indicate their potential for the future.

\section{Angular Structure}

The lunar occultation measurement on the Crab in 1964 (Bowyer et al., 1964) showed that a large part of the X-ray source from 2-10 keV was extended, with a size of about $2^{\prime}$. The pulsating component, presumably a point source, would not have been statistically significant in the data. However, it is interesting that the centroid of the distribution along the direction of motion of the moon is located within 2-3 arc sec of the pulsar, after taking into account the parallax error due to the rocket's motion downrange during its flight* (Oda et al., 1967). See Figure 1.

* The precision of that measurement is estimated to be \pm 5 arc sec. 


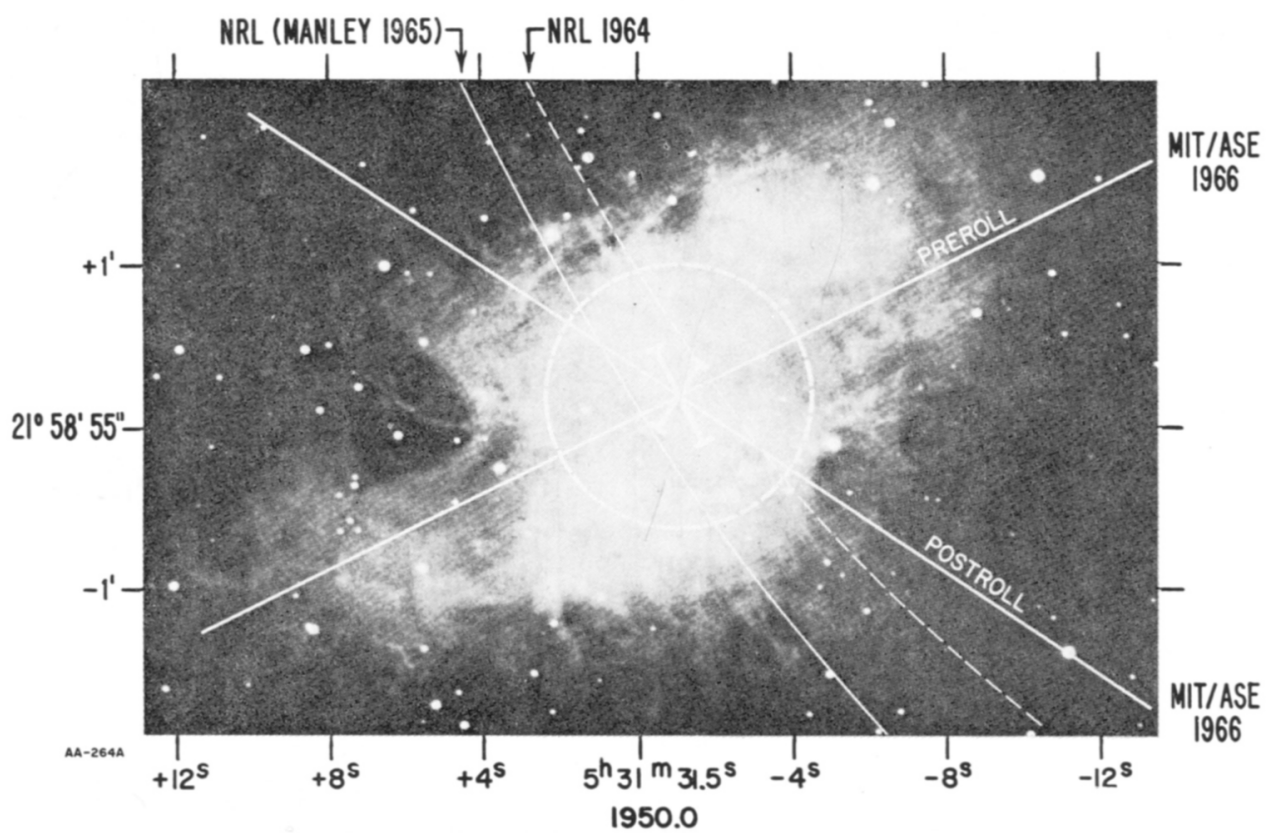

Fig. 1. Summary of correlation between visible light and X-rays from the Crab Nebula. The pulsar NP 0532 is at the origin of the coordinate system. Results from the lunar occultation of 1964 and the modulation collimator experiment of 1966 are shown, together with a visible light photo. The $100^{\prime \prime}$ diameter circle is meant to show that the best estimate of the X-ray source distribution can only give a size parameter with no very significant further information on details of the source distribution.

The MIT/ASE observation of the Crab in 1966 used a modulation collimator to scan the Crab in two directions almost perpendicular. Counting data from 1-6 keV are shown in Figure 2, along with the best fit response to an extended source. A map of this source distribution is shown in Figure 3. The centroid is located about 20" from the pulsar, one standard deviation in location. This result is consistent with the Crab source being extended, about $100^{\prime \prime}$ in diameter. It could also be oblong, or it might be a thin line extending in a southeast-to-northwest direction. In fact, there are many source configurations consistent with this result (Oda et al., 1967).

One phenomenon which could interfere with more detailed investigations of the Crab Nebula but perhaps interesting on its own is scattering of X-rays by interstellar grains. Slysh (1969) suggested that the extended source might be entirely the result of a point pulsar X-ray source whose emission is scattered into a 'halo' and loses time coherence. It appears that, among other arguments, the amount of dust required for that is too high (Naranan and Shah, 1970; Bowyer et al., 1970; Ryter, 1970). Also, measurements of the pulsar fraction at high energies where scattering is not important argue against such a picture.

A measurement of size at higher energy for the unpulsed X-ray emission would be of great interest. It might allow us to trace the structure of the magnetic field and 


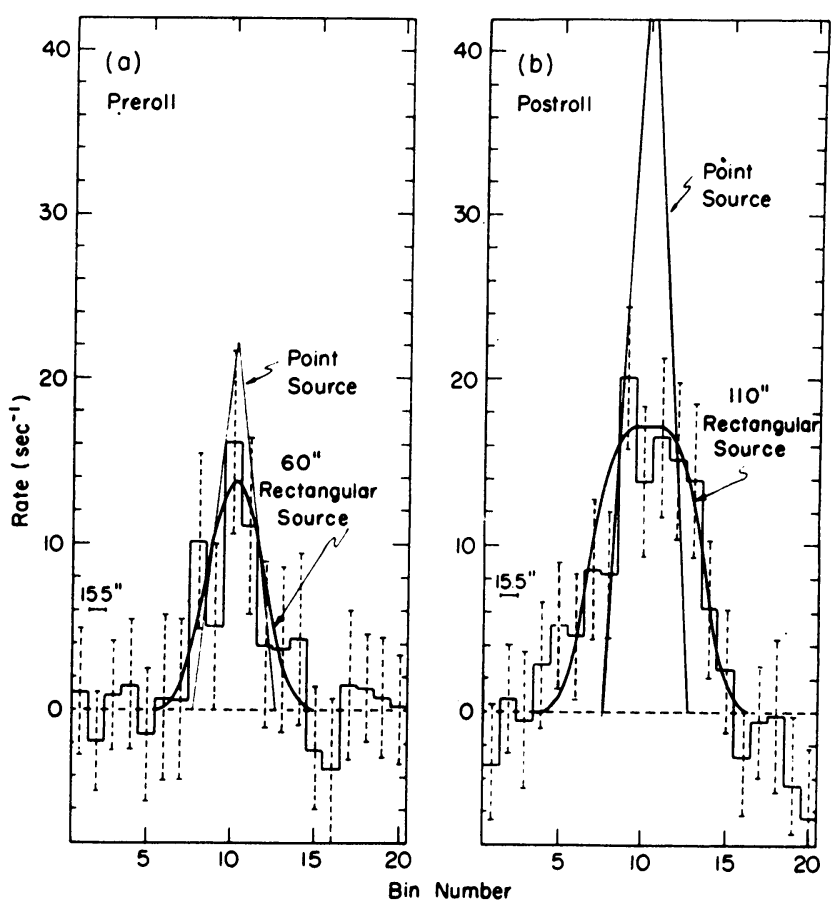

Fig. 2. Data obtained in the 1966 MIT/ASE modulation collimator experiment on the Crab. (a) and (b) are counting rate versus angle from two scans in nearly orthogonal directions across the Crab, each lasting 40 seconds. The solid lines show the count rate to be excepted from an assumed rectangular source intensity function $60^{\prime \prime}$ and $110^{\prime \prime}$ wide in the respective scan directions.

electrons in a region which may be closer and closer to the pulsar for higher energy $\mathrm{X}$-ray emission, or it may show some other result, perhaps indicating that the X-ray emission is not so directly connected with the pulsar. In a recent modulation collimator experiment, Floyd (1970) attempted to measure the Crab's size at higher energies, 25-100 keV, from a balloon. While the detector observed X-rays from the Crab, no definite modulation of the counting rate was seen with a two-grid modulation collimator of $2^{\prime}$ period. The large background count rate compared to the signal from the $\mathrm{Crab}$ in that experiment leads Floyd to interpret the results as indicating a lower limit of 1'.1 on the size. This size result is a key point in our understanding of the Crab, and should be confirmed or studied in more detail.

Future experiments in preparation and in proposal stage at AS\&E are expected to yield true images of the $\mathrm{Crab}$ in $\mathrm{X}$-rays from $\sim 0.7$ to $2 \mathrm{keV}$ using a grazing incidence telescope. A 9.5 inch diameter telescope will be flown in September 1970 on an Aerobee 170 sounding rocket. This should yield a picture with about $20^{\prime \prime}$ resolution. It will be extremely limited in contrast definition, however, due to the low efficiency of the telescope system and the short observing time available in a rocket flight. We expect to be able to distinguish major features of the source structure, however. Later versions of this instrument have been proposed, such as a 20 inch telescope for 


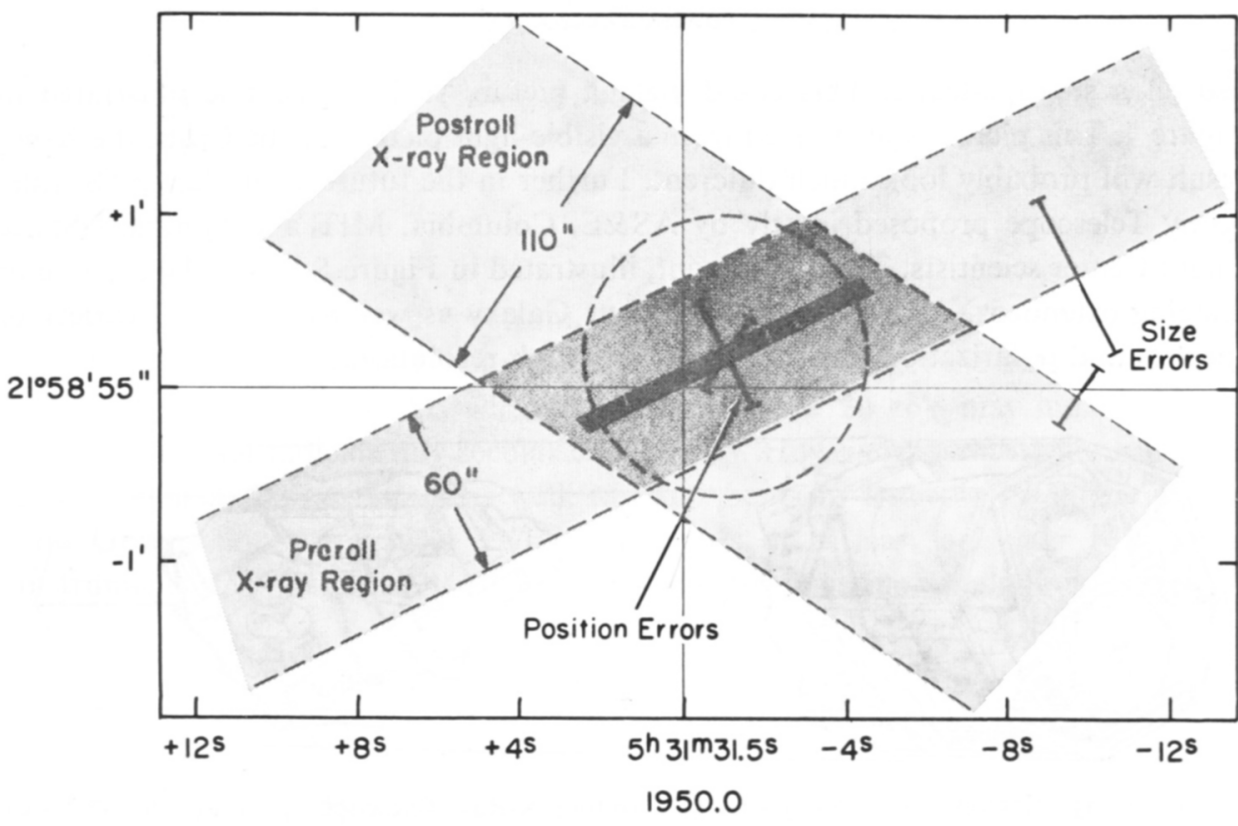

Fig. 3. X-ray source distribution indicated by the 1966 MIT/ASE experiment (Oda et al., 1967). The coordinate system is again centred on the pulsar NP 0532. The combined results of the NRL occultation in 1964 and this experiment still cannot rule out a source which is a thin line extending in a southeast-to-northwest direction, as shown in this figure.

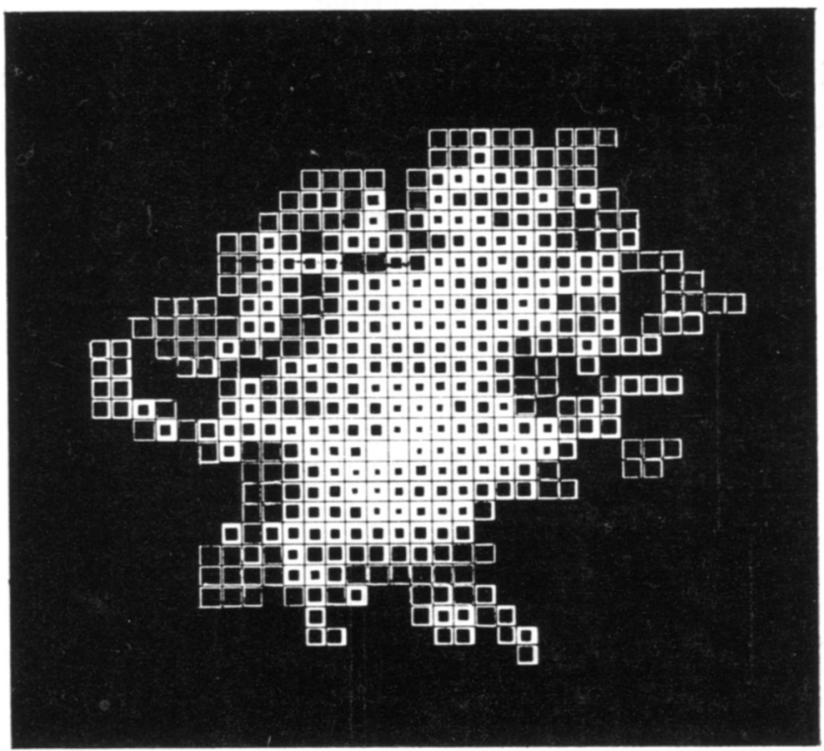

Fig. 4. Example of the type of X-ray image of the Crab Nebula to be obtained by a proposed X-ray telescope on a small satellite. The picture was derived from an optical photograph of the Crab broken into $5^{\prime \prime} \times 5^{\prime \prime}$ resolution elements. The amount of unblackened area in each resolution element is representative of the relative brightness of that element. The pulsar is located midway between the two entirely unblackened squares. 
use on a small satellite. This could yield a picture such as the one illustrated in Figure 4. This picture was derived from a visible-light picture of the Crab; the X-ray result will probably look much different. Further in the future is the Large Orbiting X-ray Telescope proposed jointly by AS\&E, Columbia, MIT and Goddard Space Flight Center scientists. This instrument, illustrated in Figure 5, would be capable of imaging extended X-ray sources outside our Galaxy as well as making a variety of spectral and polarization measurements with high resolution.

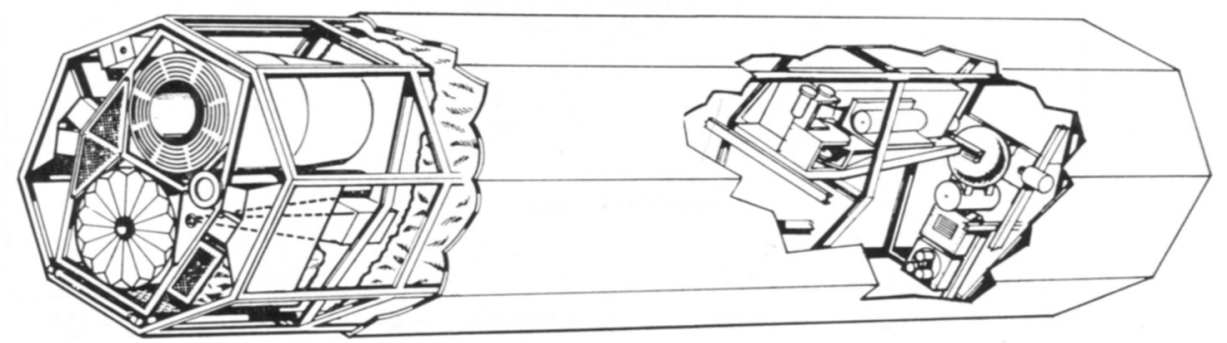

Fig. 5. X-ray telescope observatory - Large Orbiting X-Ray Telescope - proposed for NASA's pointed High Energy Astronomy Observatory satellite by AS\&E, Columbia, MIT and GSFC. Contains two telescopes, one with high resolution and the other with high efficiency, and several auxiliary instruments such as spectrometer and polarimeters. The experiment is about $8 \mathrm{ft}$ diameter by $30 \mathrm{ft}$ long.

\section{Spectrum}

A variety of observations of the Crab X-ray spectrum have been made. The sounding rocket measurements cover the energy range $0.15-20 \mathrm{keV}$ and balloon measurements

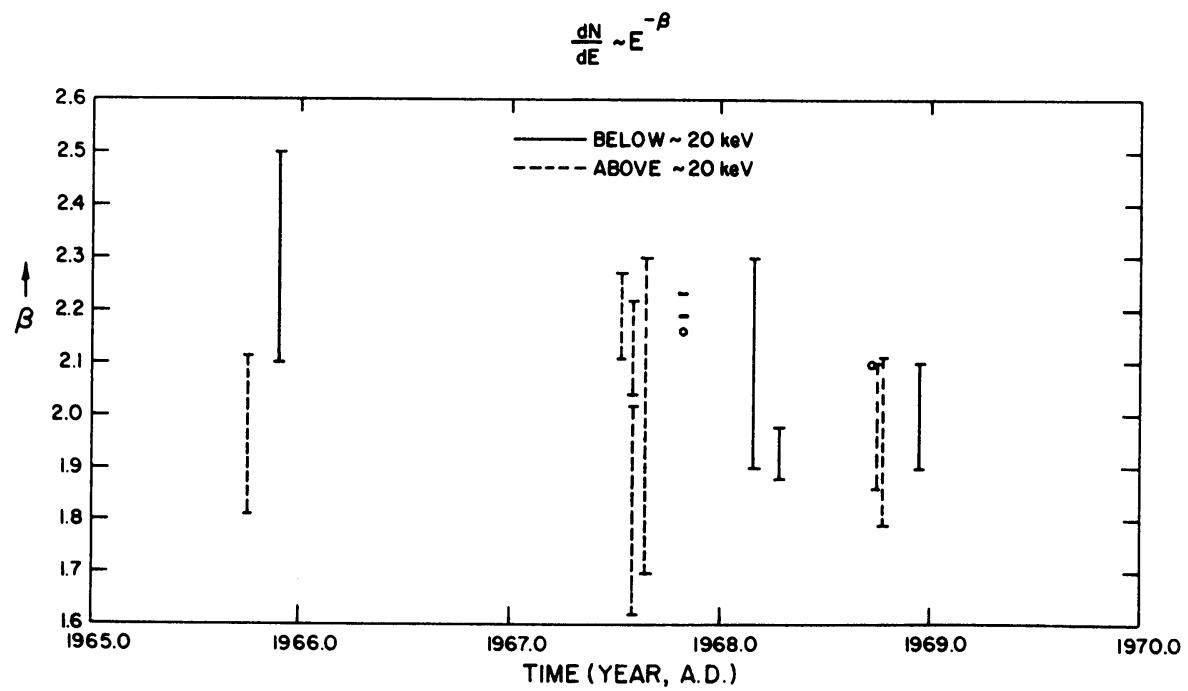

Fig. 6. Selected measurements of the Crab's X-ray spectral index. See Appendix A for references to data used in plotting this figure. 
cover the range $20-560 \mathrm{keV}$. The data above $1 \mathrm{keV}$ fit an assumed power law spectrum of the form

$$
\frac{\mathrm{d} N}{\mathrm{~d} E}=K E^{-\beta}
$$

rather than an exponential spectrum. Selected measurements of the spectral index are plotted against time in Figure 6. The scatter is fairly large; the data above $20 \mathrm{keV}$ seem to suggest close to 2.1 , whereas the data below $20 \mathrm{keV}$ may indicate $\beta \cong 2.0$. The data do not indicate any secular change in $\beta$. However, the spectral results must fit the intensity parameter, $K$ as well. Measurements by Peterson et al. $(1966,1968)$ and Gorenstein et al. $(1969,1970)$ at intervals of at least one year with similar instruments show that the intensity of the X-ray flux from the nebula remains constant

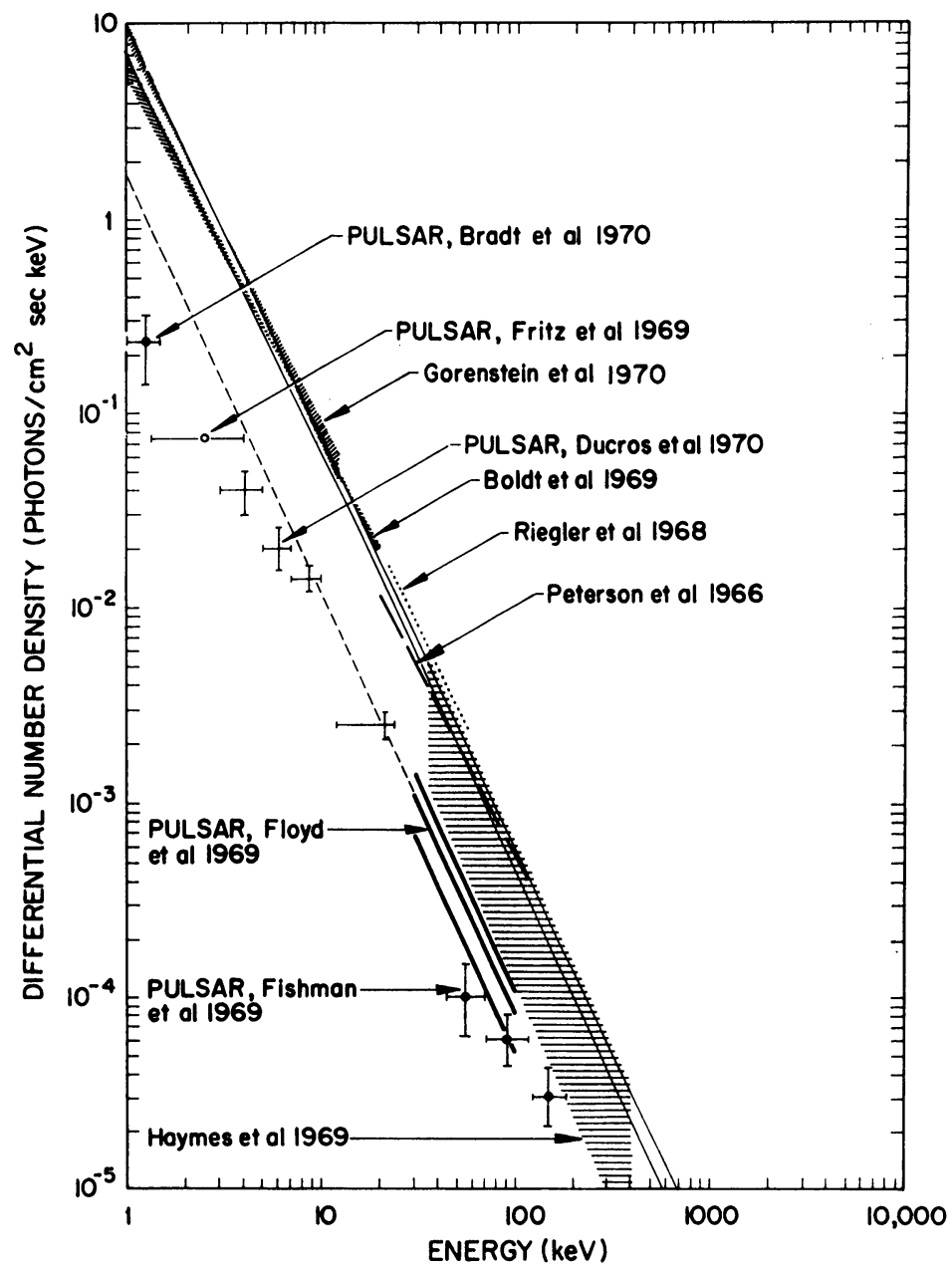

Fig. 7. X-ray spectrum of the Crab Nebula and NP 0532. 
to $5 \%$ both above and below $20 \mathrm{keV}$. If we construct a composite measured spectrum from the individual observations we obtain the results of Figure 7. A fairly close fit to the individual measurements can be obtained by a single power law

$$
\frac{\mathrm{d} N}{\mathrm{~d} E}=9 E^{-2.1} \text { photons } / \mathrm{cm}^{2} \mathrm{sec} \mathrm{keV}
$$

or by a spectrum with one break at about $20 \mathrm{keV}$

$$
\frac{\mathrm{d} N}{\mathrm{~d} E}=\left\{\begin{array}{lll}
5 & E^{-1.95} & E<20 \mathrm{keV} \\
9.1 & E^{-2.15} & E>20 \mathrm{keV}
\end{array}\right\} \text { photons } / \mathrm{cm}^{2} \mathrm{sec} \mathrm{keV}
$$

Thus, if there is a break between 1 and $500 \mathrm{keV}$, the change of index is

$$
\Delta \beta \lesssim 0.2 \text {. }
$$

The spectrum of the pulsar is also plotted in Figure 7. It appears to have about the same slope as the nebular spectrum above $10 \mathrm{keV}$, but is flatter between 1 and $10 \mathrm{keV}$. In both the case of the nebula and the pulsar, there are some discrepancies of detail between different measurements in the same energy range. However, these are not thought to be severe enough to invalidate the picture obtained in Figure 7. It is possible that future more accurate measurements may reveal a clearcut preference for a more complex nebular spectrum than a single power law. The X-ray spectrum will be compared with the optical and radio spectrum in Section 7.

\section{Interstellar Absorption}

The spectrum below $1 \mathrm{keV}$ turns over, due to interstellar absorption. Radio data on $21 \mathrm{~cm}$ absorption give a column density of $1.6 \times 10^{21}$ atoms $/ \mathrm{cm}^{2}$ to the Crab (Clark, 1965). X-ray absorption data at $1.2 \mathrm{keV}$ (Rappaport et al., 1969) set an upper limit of $N_{H} \leqslant 3 \times 10^{21}$. Measurements in the range 0.15-0.28 and 0.4-0.6 keV (Grader et al., 1970 ) find $N_{H}=1-2 \times 10^{21}$. The X-ray data are consistent with but less precise than the radio data at present.

Experiments now being designed, such as the $\mathrm{X}$-ray telescope for a small satellite proposed recently by AS\&E can obtain much more precise data on X-ray absorption. They should be able to measure interstellar abundances of certain elements, notably oxygen.

Experimental results that should be obtainable from such a telescope using an objective grating are shown in Figure 8. A spectrum like the Crab's is assumed, from a point source, and results for various column densities are shown.

\section{Polarization}

Novick and his group at Columbia have measured the Crab's polarization in X-rays 


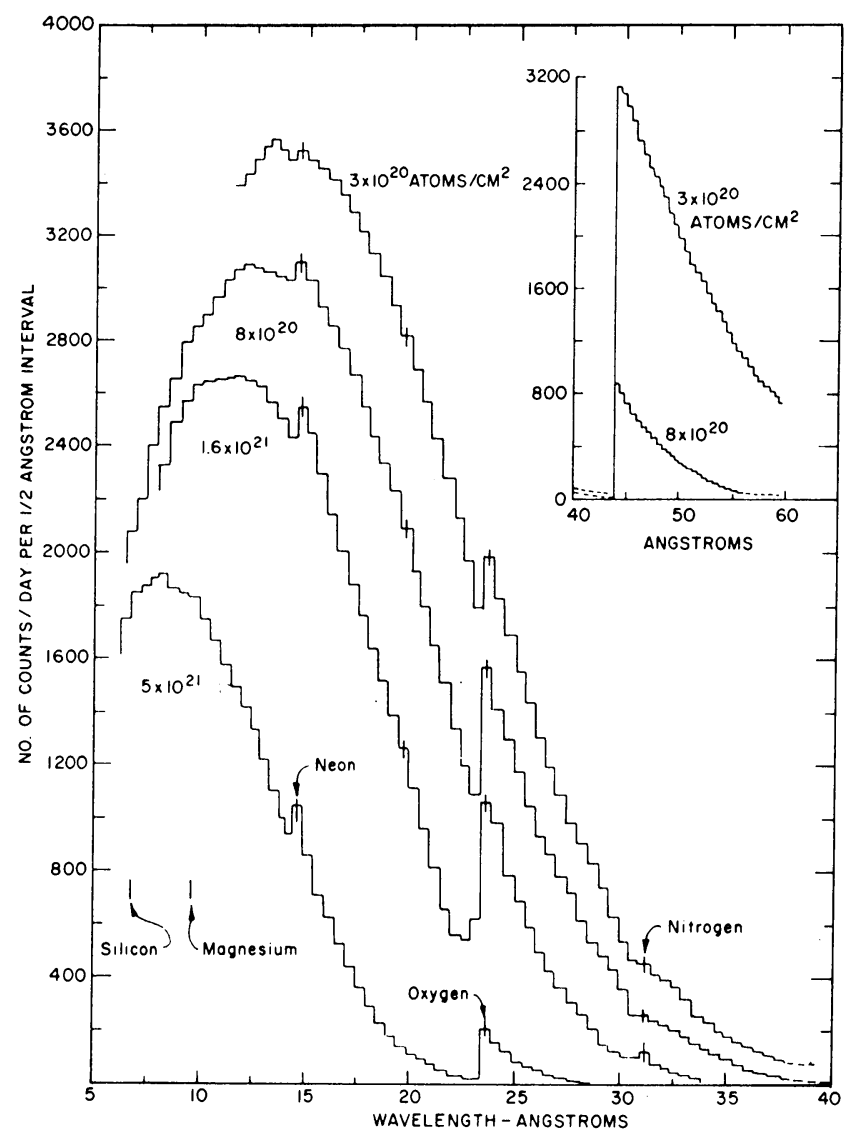

Fig. 8. Typical expected spectral data from a Small Satellite X-ray Telescope Experiment. A point source with a spectrum like the Crab's is assumed, and results are shown for various column densities of interstellar material.

to be $10 \% \pm 10 \%$. (Novick, 1969) One might expect the integrated polarization over the Crab to be of that order or less, so the present polarization data are not sufficient to make a crucial test on whether the X-rays are produced by the synchrotron process.

\section{Line Emission}

Line emission from the Crab might be possible in spite of the power law spectrum if the X-rays came from a hot gas with a complex of temperatures, densities and volumes (Sartori and Morrison, 1967). In that case, one might see emission lines. None have been found so far. Also, lines at several hundred $\mathrm{keV}$ might be present due to radioactive decay of heavy nuclides formed during the supernova explosion. The experimental upper limits set by Jacobson (1968) are a factor of four above the intensities predicted by Clayton and Craddock (1965). 


\section{Electromagnetic Spectrum of the Crab Nebula and NP 0532}

If we assume the flux density spectrum indicated by the analysis of Section 3, we can extrapolate it back to optical and radio frequencies. The result is plotted in Figure 9. The nebular X-ray spectrum with a slope of $v^{-1.1}$ meets the optical result. The intersection with the extrapolated radio spectrum occurs at about $10^{14} \mathrm{~Hz}$. The magnetic field can be derived from this, together with the known age for the nebula and is of order $10^{-4} \mathrm{G}$. The change of spectral index between the radio and $\mathrm{X}$-ray spectra is 0.84 .

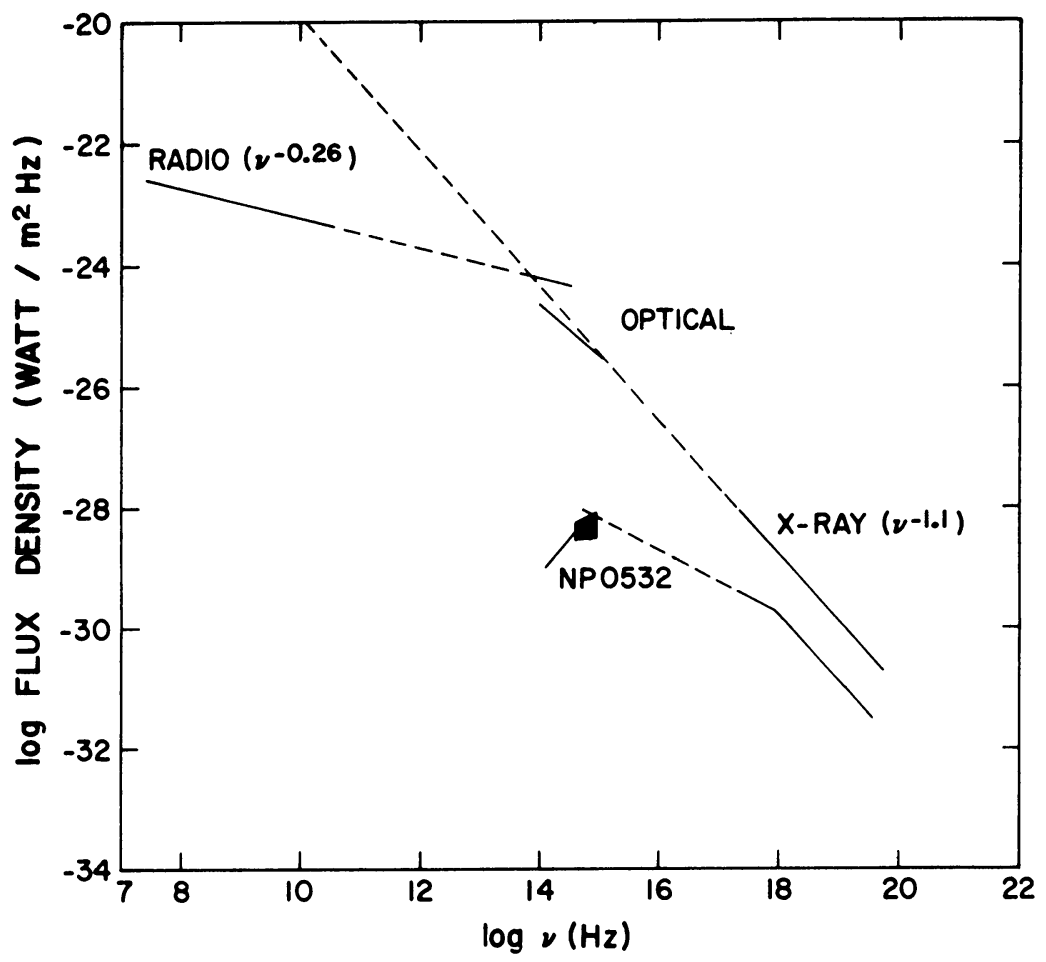

Fig. 9. Electromagnetic spectrum of the Crab.

The X-ray pulsar spectrum extrapolation to optical frequencies meets the optical data fairly well. This suggests a break in the pulsar spectrum at about $10^{18} \mathrm{~Hz}$, with a change of index of about 0.5 .

\section{X-Ray Luminosity with Size}

Recently, the AS\&E group have compared the X-ray luminosity of the Crab, Cas A and Tycho with their radio sizes (Gorenstein et al., 1970). The plot is shown in Figure 10. Once again, the Crab shows up as a singular object, having the greatest intrinsic luminosity of any galactic supernova remnant. This plot also suggests that 


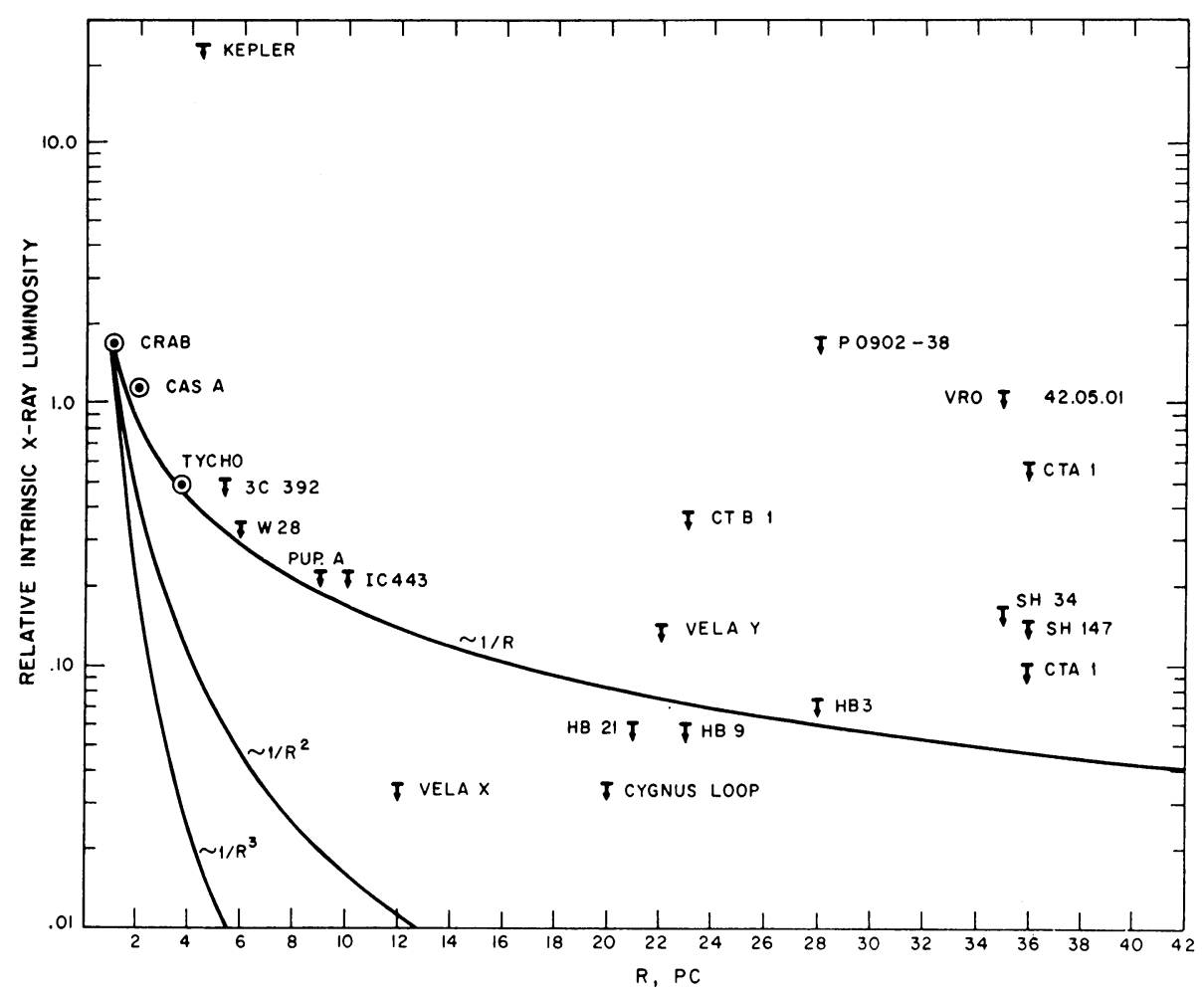

Fig. 10. X-ray luminosity versus radio diameter for several supernova remnants.

the X-ray brightness is related to size in radio. The origin of this relation, if it is confirmed by detection of weaker X-ray emission from the larger supernova remnants, is not clear.

The expansion of a magnetic field region containing high energy electrons would give decreasing luminosity with radius, if the magnetic field and electron energy decrease during expansion. A blast wave model would give increasing luminosity with radius until a peak is reached.

\section{Acknowledgements}

I wish to acknowledge helpful discussions with Wallace Tucker and Paul Gorenstein of AS\&E.

\section{References}

Boldt, E., Desai, U., and Holt, S.: 1969, Astrophys. J. 156, 427.

Bowyer, C. S., Bryam, E. T., Chubb, T. A., and Friedman, H.: 1964, Science 146, 912.

Bowyer, C. S., Mack, J., and Lampton, M.: 1970, Nature 225, 1125.

Bradt, H., et al.: 1970, Bull. Am. Phys. Soc. (in press).

Clark, B. G.: 1965, Astrophys. J. 142, 1398.

Clayton, D. D. and Craddock, W. L.: 1965, Astrophys. J. 142, 189.

Ducros, G., Ducros, R., Rocchia, R., and Tarrius, A.: 1970, Nature 227, 152.

Fishman, G. J., Harnden, F. R., Jr., and Haymes, R. C.: 1969, Astrophys. J. 156, L107. 
Floyd, F. W., Glass, I. S., and Schnopper, H. W.: 1969, Nature 224, 50.

Floyd, F. W.: 1970, Nature 226, 733.

Fritz, G., Henry, R. C., Meekings, J. F., Chubb, T. A., and Friedman, H.: 1969, Science 164, 709.

Gorenstein, P., Kellogg, E. M., and Gursky, H.: 1969, Astrophys. J. 156, 315.

Gorenstein, P., Kellogg, E. M., and Gursky, H.: 1970, Astrophys. J. 160, 199.

Grader, R. J., Hill, E. W., Seward, F. D., and Hiltner, W. A.: 1970, Astrophys. J. 159, 201.

Haymes, R. C., Ellis, D. V., Fishman, G. J., Kurfess, J. D., and Tucker, W. H.: 1968, Astrophys. J. 151, L9.

Jacobson, A. S.: 1968, Ph.D. Thesis, Univ. Calif., San Diego.

Naranan, S. and Shah, G. A.: 1970, Nature 225, 834.

Novick, R.: 1969, IAU Symp. 37, (ed. by L. Gratton).

Oda, M., Bradt, H., Garmire, G., Spada, G., Sreekantan, B. V., Gursky, H., Giacconi, R., Gorenstein, P., and Waters, J. R.: 1967, Astrophys. J. 148, 45.

Peterson, L. E., Jacobson, A. S., and Pelling, R. M.: 1966, Phys. Rev. Letters, 16, 142.

Peterson, L. E., Jacobson, A. S., Pelling, R. M., and Schwartz, D. A.: 1968, Can. J. Phys. 46, 437.

Rappaport, S., Bradt, H. V., and Mayer, W.: 1969, Astrophys. J. 157, L21.

Rappaport, S.: 1971, this symposium, Paper 2.2, p. 84.

Riegler, G. R., Boldt, E., and Serlemitsos, P.: 1968, Astrophys. J. 153, L95.

Ryter, C.: 1970, Nature 226, 1041.

Sartori, L. and Morrison, P.: 1967, Astrophys. J. 150, 385.

Slysh, V. I.: 1969, Nature 224, 159.

\section{Appendix A. Summary of Spectral Index Data Used in Figure}

\begin{tabular}{|c|c|c|c|c|}
\hline \multicolumn{4}{|c|}{$E \lesssim 20 \mathrm{keV}$ - Rocket Data } & \multirow[b]{2}{*}{$K$} \\
\hline Date & Experiment & $\Delta E \mathrm{keV}$ & $\begin{array}{l}\text { Spectral Index } \\
\left(\mathrm{d} N / \mathrm{d} E=K E^{-\beta}\right)^{*}\end{array}$ & \\
\hline $10 / 65$ & $\begin{array}{l}\text { Grader et al. } \\
\text { Science } 152 \text { (1966) } 1499\end{array}$ & $1-40$ & $2.1-2.5$ & $\sim 15$ \\
\hline $2 / 68$ & $\begin{array}{l}\text { Gorenstein et al. } \\
\text { Astrophys. J. } 156 \text { (1969) } 315\end{array}$ & $1-13$ & $1.9-2.3$ & $7.5-12.7$ \\
\hline $3 / 68$ & $\begin{array}{l}\text { Boldt et al. } \\
\text { Astrophys. J. } 156 \text { (1969) } 427\end{array}$ & $2-20$ & $1.88-1.98$ & $\sim 8-9$ \\
\hline $12 / 68$ & $\begin{array}{l}\text { Gorenstein et al. } \\
\text { Astrophys. J. } 160 \text { (1970) } 199\end{array}$ & $1-12$ & $1.9-2.1$ & $8.0-9.7$ \\
\hline \multicolumn{5}{|c|}{ photons $/ \mathrm{cm}^{2} \mathrm{sec} \mathrm{keV}$} \\
\hline \multicolumn{5}{|c|}{$E \geq 20 \mathrm{keV}$ - Data Balloon } \\
\hline & & $\Delta E \mathrm{keV}$ & $\beta$ & $K$ \\
\hline $9 / 65$ & $\begin{array}{l}\text { Peterson et al. } \\
\text { Phys. Rev. Letters } 16 \text { (1966) } 142\end{array}$ & $19-120$ & $1.81-2.11$ & 3.5 \\
\hline $6 / 67$ & $\begin{array}{l}\text { Haymes et al. } \\
\text { Astrophys. J. } 151 \text { (1968) L9 }\end{array}$ & $35-560$ & $2.11-2.27$ & $4.3-9.9$ \\
\hline $6 / 67$ & $\begin{array}{l}\text { Rocchia et al. } \\
\text { Astron. Astrophys. } 1 \text { (1969) } 48\end{array}$ & $15-100$ & $1.62-2.02$ & 7.7 \\
\hline $7 / 67$ & $\begin{array}{l}\text { Riegler, Boldt and Serlemitsos } \\
\text { Astrophys J. } 153 \text { (1968) L95 }\end{array}$ & $22-60$ & $1.7-2.3$ & 7.9 \\
\hline $7 / 67$ & $\begin{array}{l}\text { Jacobson } \\
\text { UCSD Ph D Thesis (1968) }\end{array}$ & $20-250$ & $2.04-2.22$ & \\
\hline $10 / 67$ & $\begin{array}{l}\text { Glass } \\
\text { Astrophys. J. } 157 \text { (1969) } 215\end{array}$ & $20-70$ & $\begin{array}{l}2.16 \pm ? \\
(2.19-2.23)\end{array}$ & 4.4 \\
\hline $9 / 68$ & $\begin{array}{l}\text { Rocchia et al. } \\
\text { (Preprint, 1969) }\end{array}$ & $15-100$ & $\begin{array}{l}1.86-2.10 \\
1.79-2.11\end{array}$ & $\begin{array}{l}4.8 \\
5\end{array}$ \\
\hline
\end{tabular}




\section{Discussion}

L. H. Aller: One of the most important programmes in space astronomy is to obtain observations from satellites with diffraction-limited optics. Are there any serious plans to use grazing incidence optics to obtain high resolution images of X-ray sources and spectroscopic observations?

E. M. Kellogg: The proposed large orbiting X-ray telescope will be able to take X-ray pictures with resolution of $\simeq 2$ arc sec, spectra in the X-ray range with $\lambda / \delta \lambda$ as good as $10^{4}$, and polarization measurements with angular resolution of several arc seconds, enabling an X-ray polarisation map of the Crab to be obtained.

$P$. Sanford: Would you indicate the sensitivity of your forthcoming rocket flight?

E. M. Kellogg: We can obtain about 200 counts from the entire nebula, with a limiting resolution of 10-20 arc sec, from a $190 \mathrm{sec}$ rocket observation. The grazing incidence optics, being designed originally for solar X-ray imaging, has a small effective area of $\sim 1 \mathrm{~cm}^{2}$ at $8 \AA$. The image is detected by an X-ray image intensifier which has about 50 times the sensitivity of film, which has been used for obtaining solar X-ray pictures. The next generation of rocket X-ray telescopes, available in about a year or two from now, will have ten times as much sensitivity, allowing us to obtain perhaps 2000 counts from the Crab Nebula per flight. 